\title{
SIGNIFICATION DU POLYMORPHISME ENZYMATIQUE CHEZ LES LEISHMANIES
}

\author{
A propos de trois souches hétérozygotes \\ de Leishmania infantum Nicolle, 1908, \\ Leishmania ef. tarentolae Wenyon, 1921 \\ et Leishmania aethiopica Bray, Ashford et Bray, 1973.
}

\author{
R. MAAZOUN*, G. LANOTTE*, J.-A. RIOUX*, N. PASTEUR*, \\ R. KILLICK-KENDRICK** et F. PRATLONG*
}

Collaboration technique : A. Martini-Dumas

RÉSUMÉ. L'analyse isoenzymatique de 49 souches de Leishmanies [35 Leishmania infantum Nicolle, 1908, 10 Leishmania cf. tarentolae Wenyon, 1921, 2 Leishmania tropica (Wright, 1903) et 2 Leishmania æthiopica Bray, Ashford et Bray, 1973] a révélé l'existence de structures hétérozygotes pour trois d'entre elles. Sur huit systèmes enzymatiques éprouvés : (PGM, PGI, G-6-PDH, 6-PGDH, IDH, MDH, ME et GOT), trois d'entre eux se sont exprimés par deux (PGM) ou trois (PGI et 6-PGDH) taches sur les gels d'électrophorèse. Une souche de $L$. infantum (Chien, Cévennes) et une souche de $L$. cf. tarentolae [Sergentomyia minuta (Rondani, 1843)] étaient hétérozygotes pour la PGI. Une souche de L. æthiopica (Homme, Éthiopie) était hétérozygote pour la PGI, la PGM et la 6-PGDH. Les images observées, comparables à celles obtenues avec les organismes eucaryotes à reproduction sexuée, permettent de suspecter, chez les Leishmanies, l'existence de structures diploïdes et de processus d'échanges génétiques.

\section{Significance of enzymatic polymorphism in Leishmaniae - On three heterozygous stocks of Leishmania infantum Nicolle, 1908, Leishmania ef. tarentolae Wenyon, 1921 and Leishmania athiopica Bray, Ashford and Bray, 1973.}

SUMMARY. The analysis of 8 isoenzymes (PGM, PGI, G-6-PDH, 6-PGDH, IDH, MDH, ME, GOT) of 49 stocks of leishmaniae [35 of Leishmania infantum Nicolle, 1908, 10 of Leishmania cf. tarentolae Wenyon, 1921, 2 of Leishmania tropica (Wright, 1903) and 2 of Leishmania æethiopica Bray, Ashford and Bray, 1973] revealed heterozygous structures in three stocks. These structures were expressed by two (PGM) or three (PGI, 6-PGDH) spots on the electrophoretic gels. Two stocks were heterozygous for PGI alone [one of $L$. infantum from a dog in the Cévennes, France; one of presumed Leishmania tarentolae from Sergentomyia minuta (Rondani, 1843) from P. O., France] and one was heterozygous for PGI, PGM and 6-PGDH (L. xthiopica from man in Ethiopia). The results are comparable to those seen in eukaryotic organism with sexual reproduction and suggest that leishmaniae have diploid structures and a process of genetic exchange.

* Laboratoire d'Écologie médicale et Pathologie parasitaire (Pr. J. A. Rioux), Faculté de Médecine, rue Auguste Broussonnet, F 34000 Montpellier.

** Medical Research Council (External Staff), Department of Pure and Applied Biology, Imperial College, London Sw 72 AZ (England).

Accepté le I 7 février $198 \mathrm{I}$. 
Depuis ces dix dernières années, les méthodes d'identification des Trypanosomatidae ont considérablement évolué. Aux classiques arguments morphologiques, épidémiologiques, biogéographiques et climatiques (C.A. Hoare, 1966 et 1972 ; R. Lainson et J. J. Shaw, 1979 ; R. Killick-Kendrick, 1979), s'est ajoutée une gamme très diversifiée de caractères biochimiques. Une nouvelle systématique a pris corps, s'appuyant pour la première fois sur des critères intrinsèques, critères que l'on sait indispensables à la caractérisation de tout organisme vivant. Toutefois, parmi les nouvelles techniques proposées, une seule est actuellement praticable par les laboratoires non spécialisés : l'analyse des isoenzymes. Son incontestable succès est lié à l'existence, chez les Kinetoplastida, d'un important polymorphisme qui s'exprime sous la forme de variants facilement révélés, comme autant d'électromorphes, par les techniques courantes. Les différents électromorphes de même activité catalytique (ou isoenzymes) peuvent être codés par un ou plusieurs gènes. L'analyse conjointe d'un nombre suffisant de systèmes enzymatiques, judicieusement choisis, permet de caractériser chaque souche par ses zymogrammes. Chez les Leishmanies, le mode de reproduction asexué garantit la stabilité des zymogrammes tant qu'aucune mutation n'intervient. Les souches et, a fortiori, les clones possédant les mêmes zymogrammes sont considérés comme appartenant à la même unité taxonomique élémentaire. Le concept de zymodème (D. G. Godfrey, 1979) s'applique précisément à ce type d' " unité ».

Toutefois, l'utilisation de ce concept à des fins systématiques, voire phylétiques réclame une certaine prudence, en raison des indications de plus en plus nombreuses suggérant l'existence d'une diploïdie, voire d'échanges génétiques. Ainsi, dans un article très documenté sur le sous-genre Trypanozoon, W. C. Gibson et al. (1980) présentent plusieurs illustrations évoquant, de manière troublante, les hétérozygotes observés chez les organismes eucaryotes diploïdes. Les deux types classiques d'électromorphes, à deux (monomères) ou trois (dimères) taches, y sont clairement figurés. Bien plus, en étudiant les isozymes de 17 souches sympatriques de Trypanosoma brucei brucei, A. Tait (1980) constate que les proportions d'homozygotes et d'hétérozygotes se répartissent selon la loi de Hardy-Weinberg. L'auteur conclut à l'existence de recombinaisons génétiques au sein d'une population mendélienne, c'est-àdire diploïde et à reproduction sexuée.

Or, jusqu'à présent, malgré la qualité et le nombre des travaux réalisés (P. J. Gardener et al. 1973 et 1974 ; M. L. Chance et P. J. Gardener, 1975 ; M. Al-Taqi et D. A. Evans, 1978 ; M. B. Rassam et al., 1979; T. I. Aljeboori et D. A. Evans, 1980 a et b ; J. A. Rioux et al., 1980 ; R. Maazoun et al., 1981) l'existence de structures « hétérozygotes » n'a pas été mentionnée chez les Leishmanies. C'est précisément de la réalité de telles structures, observées en France et en Éthiopie et de leur signification dont il est question dans le présent article.

\section{Matériel et méthode}

L'analyse a porté sur un total de 49 souches isolées (tableau I) de :

1) 34 cas de leishmaniose viscérale canine, originaires des Cévennes méridionales. 
Ces souches ont été obtenues par ponction ganglionnaire, lors d'enquêtes systématiques (G. Lanotte et al., 1979) ou d'examens vétérinaires à but diagnostique. Il s'agit dans tous les cas de Leishmania infantum Nicolle, 1908 ;

2) 9 cas de leishmaniose généralisée du Gecko Tarentola mauritanica (L., 1758), provenant des environs de Banyuls-sur-Mer (Pyrénées-Orientales). Les cultures sur NNN ont été obtenues par ponction cardiaque. Il s'agit de Leishmania tarentolae Wenyon, $1921^{1}$. Dans le même site, une souche a été obtenue par culture du contenu intestinal de Sergentomyia minuta (Rondani, 1843), vecteur habituel du parasite ;

TABlEAU I. - Liste des souches étudiées pour les huit systèmes enzymatiques : PGM, PGI, G-6-PDH, 6-PGDH, IDH, MDH, ME et GOT. Sur 49 souches, seules 3 d'entre elles (LEM 47, LEM 87 et LEM I09) présentent une ou plusieurs structures hétérozygotes.

\begin{tabular}{llll}
\hline No de référence & Taxon & $\begin{array}{c}\text { Hôte } \\
\text { (Détermination } \\
\text { clinique) }\end{array}$ & $\begin{array}{c}\text { Origine } \\
\text { géographique }\end{array}$ \\
\hline LEM 75 & $\begin{array}{l}\text { Leishmania } \\
\text { infantum }\end{array}$ & $\begin{array}{l}\text { Enfant } \\
\text { (leishmaniose } \\
\text { viscérale) }\end{array}$ & $\begin{array}{l}\text { France } \\
\text { (Cévennes) }\end{array}$ \\
\hline
\end{tabular}

LEM $35,47,48,49$,

$50,53,54,55,57$,

$59,60,63,64,65$,

$68,72,79,80,81$,

$86,88,89,90,91$,

$92,93,98,99,102$,

Leishmania

infantum

$103,104,105,106$,

107

\begin{tabular}{llll}
\hline $\begin{array}{l}\text { LEM 112, 113, 115, } \\
123,124\end{array}$ & $\begin{array}{l}\text { Leishmania } \\
\text { tarentolae }\end{array}$ & $\begin{array}{l}\text { Tarentola } \\
\text { mauritanica } \\
\text { (leishmaniose } \\
\text { générale) }\end{array}$ & $\begin{array}{l}\text { France } \\
\text { (Pyrénées- } \\
\text { Orientales) }\end{array}$ \\
\hline LEM 87 & $\begin{array}{l}\text { Leishmania } \\
\text { cf. tarentolae }\end{array}$ & $\begin{array}{l}\text { Sergentomyia } \\
\text { minuta } \\
\text { (localisation } \\
\text { digestive) }\end{array}$ & $\begin{array}{l}\text { France } \\
\text { (Pyrénées- } \\
\text { Orientales) }\end{array}$ \\
\hline LEM 109, 144 & $\begin{array}{l}\text { Leishmania } \\
\text { ethiopica }\end{array}$ & $\begin{array}{l}\text { Homme } \\
\text { (leishmaniose } \\
\text { cutanée) }\end{array}$ & Éthiopie \\
\hline LEM 143 & $\begin{array}{l}\text { Leishmania } \\
\text { tropica }\end{array}$ & $\begin{array}{l}\text { Homme } \\
\text { (leishmaniose } \\
\text { cutanée) }\end{array}$ & Éthiopie \\
\hline LEM I4I & $\begin{array}{l}\text { Leishmania } \\
\text { tropica }\end{array}$ & $\begin{array}{l}\text { Chien } \\
\text { (leishmaniose } \\
\text { cutanée) }\end{array}$ & Inde \\
\hline
\end{tabular}

Chien

(leishmaniose France

viscérale) (Cévennes)

I. Cette espèce a été signalée pour la première fois en France à Banyuls-sur-Mer, dans les Pyrénées-Orientales (J. A. Rioux et coll., r969) puis retrouvée dans la même localité dix ans plus tard (J. A. Rioux et coll., 1979). 
3) 2 cas de leishmaniose cutanée humaine à Leishmania athiopica Bray, Ashford et Bray, 1973, originaires d'Éthiopie.

4) 2 cas de leishmaniose cutanée à Leishmania tropica (Wright, 1903), dont un humain d'origine éthiopienne, l'autre canin d'origine indienne.

5) 1 cas de leishmaniose infantile autochtone (Cévennes) à Leishmania infantum Nicolle, 1908. Cette souche (LEM 75) a servi de référence.

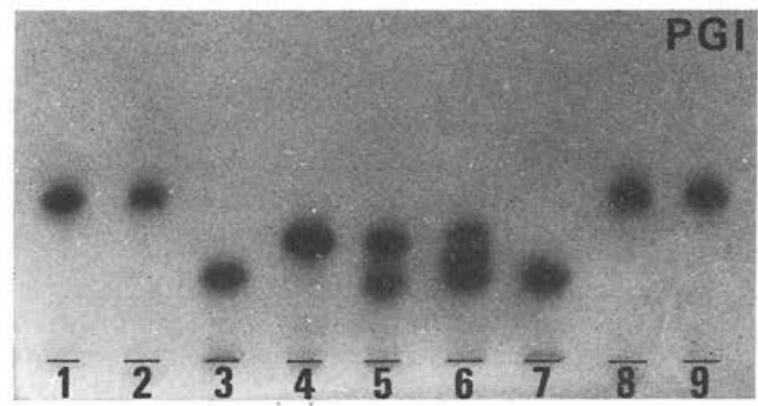

a

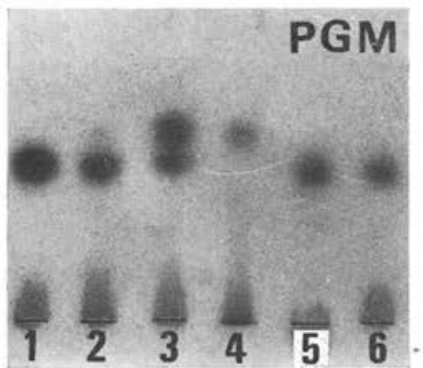

b

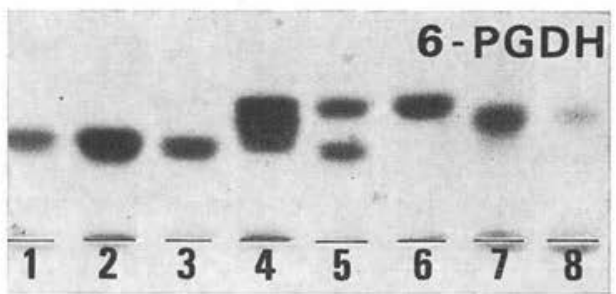

C

FIG. I.

a) PGI : Phosphoglucose isomérase. Êlectrophorèse en gel épais. I, 2, 8 et 9: L. infantum (LEM 75. LEM 104, LEM Io3 et LEM 102). 3 et $7:$ L. tropica (LEM I43 et LEM I4I). 4 et $6:$ L. athiopica (LEM I44 et LEM I09). 5: L. tropica (LEM I43) et L. athiopica (LEM I44). Pour L. athiopica (LEM rog), l'activité enzymatique se traduit par un zymogramme à trois bandes régulièrement espacées, la médiane la plus intense (6). Dans le cas particulier (système dimérique), cette image est caractéristique d'une structure hétérozygote. Le mélange extemporané (5) de L. tropica (LEM I43) et L. athiopica (LEM I44) se traduit par l'addition des bandes propres à chaque zymodème : aucun échange ne s'est produit.

b) PGM : Phosphoglucomutase. Électrophorèse en gel épais. 1, 2, 5 et $6: L$ tropica (LEM I43). 3 et 4 : L. athiopica (LEM rog et LEM r44). Pour L. athiopica, hétérozygote (LEM ro9), la Phosphoglucomutase, monomérique, s'exprime par un zymogramme à deux bandes.

c) 6-PGDH : 6-Phosphogluconate déshydrogénase. Électrophorèse en gel épais. I et $2: L$. infantum (LEM IO2 et LEM I04). 3: L. tropica (LEM 143). 4 et 6: $L$. athiopica (LEM rog et LEM I44). 5: mélange extemporané de L. tropica (LEM 143) et L. athiopica (LEM I44). Pour L. athiopica hétérozygote (LEM I09), la 6-Phosphogluconate déshydrogénase, dimérique comme la Phosphoglucose isomérase, présente un zymogramme à trois bandes (4). Le mélange extemporané (5) des extraits de L. tropica (LEM I43) et L. athiopica (LEM I44) monomorphes (3 et 6), se traduit par la simple addition des bandes respectives des deux souches. 
Les extraits sont préparés selon la technique décrite par R. Maazoun et al. (1981). L'électrophorèse est réalisée en gel d'amidon épais $(1 \mathrm{~cm})$ et mince $(0,1 \mathrm{~cm})$ à partir d'échantillons conservés dans l'azote liquide sous forme de perles de $200 \mu \mathrm{l}$. 8 systèmes enzymatiques sont étudiés :

- la Phosphoglucomutase (PGM) E.C.2.7.5.1.

- la Phosphoglucose isomérase (PGI) E.C.5.3.1.9.

- la Glucose-6-Phosphate déshydrogénase (G-6-PDH) E.C.1.1.1.49.

- la 6-Phosphogluconate déshydrogénase (6-PGDH) E.C.1.1.1.44.

- l'Isocitrate déshydrogénase (IDH) E.C.1.1.1.42.

- la Malate déshydrogénase (MDH) E.C.1.1.1.37.

- l'Enzyme malique ou décarboxylase (ME) E.C.1.1.1.40.

- la Glutamate-oxaloacétate transaminase (GOT) E.C.2.6.1.1.

\section{Résultats et commentaires}

Sur les 8 systèmes éprouvés (tableau II), Leishmania infantum (souche canine d'origine cévenole, LEM 47) et Leishmania cf. tarentolae (souche isolée de Sergentomyia minuta, Pyrénées-Orientales, LEM 87) révèlent une structure hétérozygote pour un seul d'entre eux, la Phosphoglucose isomérase (PGI). Trois bandes régulièrement espacées, la médiane la plus intense, témoignent de la présence d'une protéine dimère (fig. 1 et 2, tableau II). Leishmania athiopica (LEM 109) possède au moins 3 systèmes hétérozygotes (tableau II) : la Phosphoglucose isomérase (PGI) et la 6-Phosphogluconate déshydrogénase (6-PGDH), toutes deux dimériques, s'expriment par trois bandes; la Phosphoglucomutase (PGM), monomérique, n'offre que deux bandes (fig. 3).

TABLeAu II. - Fréquence du polymorphisme chez les 3 souches hétérozygotes de Leishmania infantum (LEM 47), Leishmania cf. tarentolae (LEM 87) et Leishmania athiopica (LEM ro9).

\begin{tabular}{llc}
\hline $\begin{array}{c}\text { No de } \\
\text { Référence }\end{array}$ & \multicolumn{1}{c}{ Systèmes hétérozygotes } & $\begin{array}{c}\text { Fréquence de } \\
\text { l'hétérozygotie }\end{array}$ \\
\hline LEM 47 & Phosphoglucose isomérase (PGI) & $1 / 8$ \\
\hline LEM 87 & Phosphoglucose isomérase (PGI) & $1 / 8$ \\
\hline LEM 109 & $\begin{array}{l}\text { Phosphoglucomutase (PGM) } \\
\text { Phosphoglucose isomérase (PGI) }\end{array}$ & $3 / 8$ \\
\hline
\end{tabular}

Au surplus, le mélange extemporané des extraits de deux souches (LEM 143 et LEM 144 : leishmanioses cutanées d'origine éthiopienne) donne un zymogramme 
correspondant à la simple juxtaposition de leurs bandes respectives ( fig. 2) ; autrement dit, ce zymogramme diffère de celui de Leishmania athiopica hétérozygote (LEM 109) par l'absence de bande médiane.

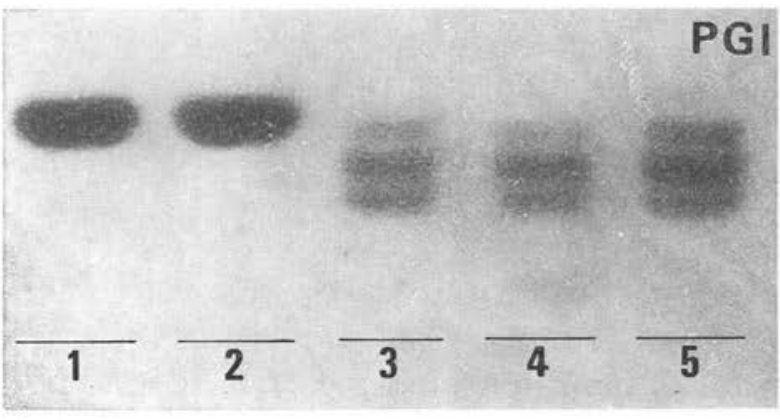

a

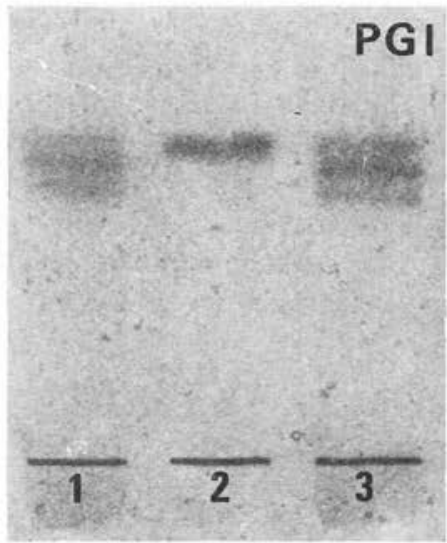

b

FIG. 2. - PGI : Phosphoglucose isomérase. Électrophorèse en gel mince. a) I et 2 : L. tropica (LEM I4I et LEM I 43). 3, 4 et $5:$ L. athiopica (LEM IO9); b) I et $3: L$. cf. tarentolae (LEM 87). 2 : L. tarentolae (LEM II9). Pour cette enzyme, dimérique, L. athiopica (LEM Iog) et $L$. cf. tarentolae (LEM 87) présentent trois bandes (a 3,4 et 5 ; b I et 3 ) caractéristiques d'une structure hétérozygote. Les souches homozygotes (a I et $2 ; b_{2}$ ) ne s'expriment que par une seule tache.

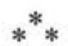

$\mathrm{Au}$ demeurant, si l'analyse enzymatique suggère l'existence de structures hétérozygotes comparables à celles des eucaryotes diploïdes, leur signification et les mécanismes de production restent, pour l'instant, du domaine des hypothèses :

1) A partir d'un matériel habituellement haploïde, on peut concevoir un processus de duplication génique suivi de mutation : deux gènes d'un même système enzymatique, situés sur le même chromosome, coderaient donc pour deux allozymes différentes. Dans cette éventualité, la plupart des gènes existeraient à un seul exemplaire dans chaque individu et leur produit apparaitrait sous la forme d'une tache unique sur les gels d'électrophorèse. Quelques gènes dupliqués pourraient, alors, produire des zymogrammes typiques d'hétérozygotes diploïdes.

2) On peut également imaginer la production de variants enzymatiques par " mutations somatiques " au sein de dèmes diploïdes, mais asexués.

3) En fait, le nombre important de gènes hétérozygotes, souvent observés dans une même souche, l'équilibre statistique de phénotypes enzymatiques, constaté dans certaines populations de Trypanosomes (A. Tait, loc. cit.), l'obtention expérimentale de souches intermédiaires par infestation mixte des vecteurs (V. M. Safyanova et A. N. Alexeiev, 1977) constituent autant d'arguments en faveur d'une diploidie et d'un processus de multiplication sexuée. 


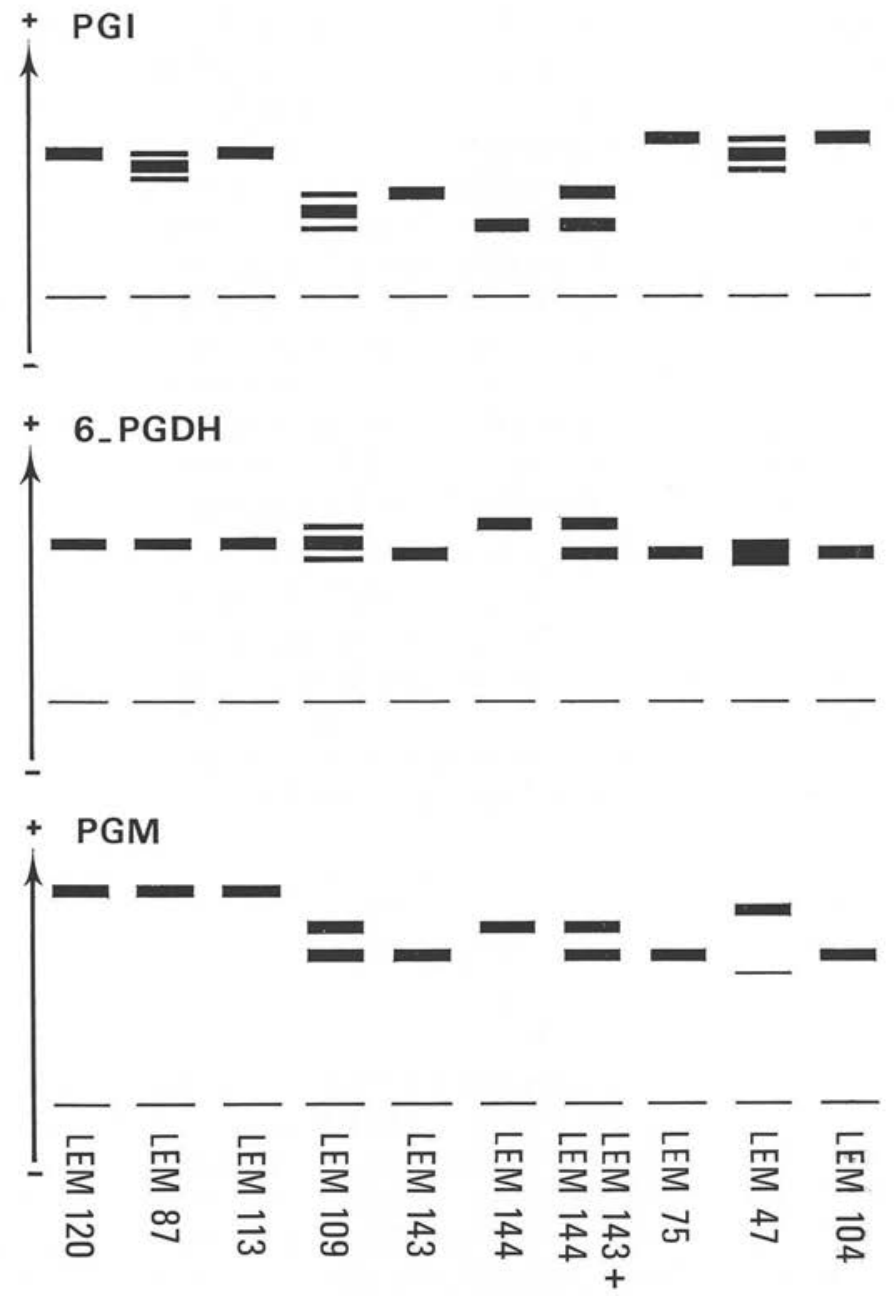

FIG. 3. - Leishmania cf. tarentolae (LEM 87) présente une structure hétérozygote pour la PGI. L. athiopica (LEM IO9) présente une telle structure pour la PGI (dimère), la 6-PGDH (dimère) et la PGM (monomère). L. infantum (LEM 47) présente une structure hétérozygote nette pour la PGI. Une épaisseur inhabituelle de la bande 6-PGDH traduit peut-être, une structure hétérozygote. Le mélange extemporané des deux souches monomorphes $L$. athiopica (LEM I44) et L. tropica (LEM I43) ne donne que deux bandes pour la PGI et la 6PGDH : aucun processus d'hybridation ne s'est produit in vitro. 
Toutefois, dans cette hypothèse, il reste à rendre compte de la grande proportion de loci homozygotes observée dans la plupart des cas. Remarquons cependant que de telles dispositions, quoique rares, se rencontrent chez les organismes diploïdes à reproduction strictement sexuée (Rongeurs du genre Dipodomys, W. E. Johnson et R. K. Selander, 1971) et il n'est pas impossible que les Trypanosomatidae dont l'originalité physiologique est déjà très grande, appartiennent à ce groupe d'organismes. La basse fréquence du polymorphisme pourrait également être le résultat d'une forte pression de sélection exercée par les divers hôtes sur des populations parasitaires à taux de renouvellement élevé (P. J. Génermont, 1980). On ne peut enfin éliminer le biais introduit par les épidémiologistes dans l'échantillonnage des souches : les isolats les plus nombreux proviennent actuellement de l'Homme ou des Mammifères commensaux, alors que les processus sexués pourraient se dérouler ailleurs, chez les vecteurs (R. Killick-Kendrick, 1979) voire chez les Vertébrés sauvages. Dans cette dernière éventualité, le monomorphisme enzymatique pourrait être induit par les " hôtes secondaires " (sensu P.C.C. Garnham, 1965) et, par conséquent, s'observerait essentiellement dans les cycles artificialisés.

En toute logique, si l'existence de la sexualité était confirmée, le concept de zymodème devrait être étendu au complexe des phénotypes issus d'une même population mendélienne, c'est-à-dire, à un ensemble de zymogrammes différents. Cependant, en raison du monomorphisme habituel des souches isolées, l'acception initiale pourrait être provisoirement maintenue. Le zymodème continuerait ainsi à servir d" "unité opérationnelle" dans les travaux de taxonomie numérique qui, rappelons-le, ne font appel à aucune hypothèse biologique.

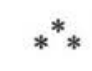

Quoi qu'il en soit, les considérations théoriques, développées à l'occasion de ce travail, renforcent singulièrement l'intérêt porté par les écologistes à la connaissance biochimique des Trypanosomatidae. Ainsi, l'analyse enzymatique des Leishmanies, réalisée sur des "populations de souches", et dans le double esprit, génétique et épidémiologique, devrait conduire à une meilleure connaissance des phénomènes de reproduction et de circulation des parasites dans les réservoirs et les vecteurs. L'approche "systémique ", structurale et fonctionnelle, des foyers leishmaniens en bénéficierait largement.

Remerciements. Nos plus vifs remerciements vont à Messieurs R. W. Ashford, R. S. Bray, M. L. Chance, J. David, P. C. C. Garnham, P. J. Génermont, D. G. Godfrey et $\mathrm{W}$. Peters qui nous ont très aimablement communiqué certaines souches et nous ont fait largement profiter de leur expérience. 


\section{BIBLIOGRAPHIE}

AlJEBOoRI T. I., Evans D. A. : Leishmania spp. in Iraq. Electrophoretic isoenzyme patterns. I. Visceral leishmaniasis. Trans. R. Soc. Trop. Med. Hyg., I980 a, 74, I69-1 77 .

AlJEBoori T. I., Evans D. A. : Leishmania spp. in Iraq. Electrophoretic isoenzyme patterns. II. Cutaneous leishmaniasis. Trans. R. Soc. Trop. Med. Hyg., I980 b, 74, I78-187.

AL-TAOI M., Evans D. A. : Characterization of Leishmania spp. from Kuwait by isoenzyme electrophoresis. Trans. R. Soc. Trop. Med. Hyg., I978, 72, 56-65.

Chance M. L., GARdener P. J. : Biochemical and morphological differentiation within the genus Leishmania. Trans. R. Soc. Trop. Med. Hyg., 1975, 69, 9.

Chance M. L., Schnur L. F., Thomas S. C. : The identity of African rodent leishmanias. Trans. R. Soc. Trop. Med. Hyg., 1977, 7I, II 3.

Evans D. A. : Kinetoplastida. In : Methods of cultivating parasites in vitro. Acad. Press, 1978, 55-88.

Gardener P. J., Chance M. L., Peters W. : Isoenzyme variation between life-cycle stages of Leishmania. Trans. R. Soc. Trop. Med. Hyg., 1973, 67, 23.

Gardener P. J., Chance M. L., Peters W. : Biochemical taxonomy of Leishmania. II : Electrophoretic variation of malate dehydrogenase. Ann. Trop. Med. Parasit., 1974, 68, 31 7-325.

GaRnham P. C. C. : The Leishmanias, with special reference to the role of animal reservoirs. $\mathrm{Am}$. Zoologist, I965, S, I 4 I-I 5 I.

GÉNERMONT P. J. : Les animaux à reproduction uniparentale. In : Les problèmes de l'espèce dans le règne animal. Soc. zool. Fr. Ed., I980, 3, 287-320.

Gibson W. C., MARshall DE C. T. F., GoDFrey D. G. : Numerical analysis of enzyme polymorphism : A new approach to the epidemiology and taxonomy of Trypanosomes of the subgenus Trypanozoon. In : Advances in Parasitology, Acad. Press, 1980, 18, 176-246.

Godfrey D. G. : The zymodemes of Trypanosomes. Symp. Br. Soc. Parasit., 1979, 17, 31-53.

HoARE C. A. : The classification of mammalian Trypanosomes. Ergebn. Mikrobiol. ImmunForsch. Exper. Ther., I966, 43-57.

HoARE C. A. : The Trypanosomes of Mammals. Blackwell Sc. Pub., r972, 749 p.

Johnson W. E., SELANDER R. K. : Protein variation and systematics in Kangaroon Rats (genus : Dipodomys). Systematic Zoology, 1971, 20, 377-405.

KILLICK-KENDRICK R. : Biology of Leishmania in Phlebotomine sandflies. In : Biology of the Kinetoplastida, Acad. Press, 1979, 2, 395-460.

Larnson R., SHAw J. J. : The role of animals in the epidemiology of South American Leishmaniasis. In : Biology of the Kinetoplastida, Acad. Press, 1979, 2, I-I 6.

Lanotte G., Rioux J. A., PÉrières J., Vollhardt Y. : Écologie des Leishmanioses dans le sud de la France. Io. Les formes évolutives de la leishmaniose viscérale canine. Élaboration d'une typologie bio-clinique à finalité épidémiologique. Ann. Parasit. Hum. Comp., 1979, 54, 277-

Maazoun R., Lanotre G., Pasteur N., Rioux J. A., Kennou M. F., Pratlong F. : Écologie des Leishmanioses dans le sud de la France. I6. Contribution à l'analyse chimiotaxonomique des parasites de la leishmaniose viscérale méditerranéenne. A propos de 55 souches isolées en Cévennes, Côte d'Azur, Corse et Tunisie. Ann. Parasit. Hum. Comp., I981, s6, I3I-I46.

MAYr E. : Population, espèces et évolution. Herman, 1974, 496 p.

Rassam M. B., Al-Mudhaffar S. A., Chance M. L. : Isoenzyme characterization of Leishmania species from Iraq. Ann. Trop. Med. Parasit., 1979, 73, 527-534.

Ready P. D., Mrles M. A. : Delimitation of Trypanosoma cruzi zymodemes by numerical taxonomy. Trans. R. Soc. Trop. Med. Hyg., I979, 74, 238-24I.

Rioux J. A., KIllick-Kendrick R., GARnham P. C. C. : Leishmania tarentolae and other blood parasites of geckoes in the south of France. Trans. R. Soc. Trop. Med. Hyg., I979, 73, 319.

Rioux J. A., KNoepfler L. P., Martini A. : Présence en France de Leishmania taventolae Wenyon, I92I, parasite du Gecko Tarentola mauritanica (L. 1758). Ann. Parasit. Hum. Comp., 1969, 44, I I $5-116$.

Rioux J. A., Lanotte G., Maazoun R., Perello R., Pratlong F. : Leishmania infantum Nicolle, I9o8, agent du bouton d'Orient autochtone. A propos de l'identification biochimique de deux souches isolées dans les Pyrénées-Orientales. C. R. Acad. Sc. Paris, 1980, 291, 701-703.

Safyanova V. M., Alexerev A. N. : Mixed experimental infection of Phlebotomus papatasi (Sc.) with different species of Leishmania. In : Écologie des Leishmanioses. Colloques int. C.N.R.S., $1977, \mathrm{n}^{\circ} 239, \mathrm{I} 53^{-1} 56$.

TAIT A. : Evidence for diploidy and mating in Trypanosomes. Nature, r980, 287, 536-538. 